\title{
Numerical Solution of a Single-Phase Three-Dimensional Flow in Porous Media by the Hybrid Mixed Finite Element Method
}

\author{
Joel Antonio G. de Moraes, Eduardo C. de Abreu, Paola C. Ferraz
}

\begin{abstract}
The objective of this work is the study of numerical approximation for solving the problem of single phase Darcy flow. The mathematical model is given in three space dimension by one scalar partial differential equation of elliptic nature. Mixed and hybrid finite element method is used for discretization of the model, resulting in an algebraic linear system. We present good approximations of the three-dimensional problem at hand.
\end{abstract}

Key words:

Flow in Porous Media, Hybrid Mixed Finite Element Method, Darcy's Flow

\section{Introduction}

The focus of this work is the numerical approximation of differential problems involving partial differential equations of elliptic nature, in the context of modeling in fluid dynamics in porous media. Particularly, we want to obtain a numerical solution of the model associated to the incompressible flow of single-phase fluids in a threedimensional porous media. This flow obeys the Darcy's law, which relates the volumetric flux (the Darcy's velocity) of the fluid with the pressure gradient. The governing equation of this problem is

$$
\begin{aligned}
& u(x)=-K(x) \cdot \nabla p(x), x \in R^{3} \\
& \nabla . u(x)=f,
\end{aligned}
$$

$\mathrm{u}$ is the Darcy's velocity, $\mathrm{K}$ is the permeability tensor, that can be homogeneous or heterogeneous, $p$ is the pressure and $f$ is the source term. Boundary conditions are necessary to complete the mathematical problem.

We used the hybrid mixed finite element ${ }^{1,3}$ (HMFEM) to discretize the elliptic problem, together with a domain decomposition technique and the lowest order RaviartThomas as approximation space. The HMFEM simultaneously approximates the velocity field and the pressure. Also, the HMFEM guarantees the local conservation of the flux ${ }^{2}$ and is known to provide good approximation for the Darcy's velocity ${ }^{2,3}$.

After the discretization, we obtain an algebraic linear system of equations with a symmetric positive-definite matrix, solved by an appropriate numerical method. Numerical experiments are presented and discussed.

\section{Results and Discussion}

We performed simulations for the $1 / 4$ of five spot problem (1) with the boundary conditions in Figure 1.

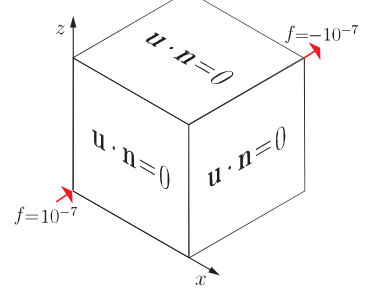

Figure 1. Boundary conditions and source term.

These simulations show that the hybrid mixed finite element method with the lowest order Raviart-Thomas space provides a very good approximation of the threedimensional single-phase incompressible flow problem. The method is able to capture the solution profile of both the velocity and pressure fields.

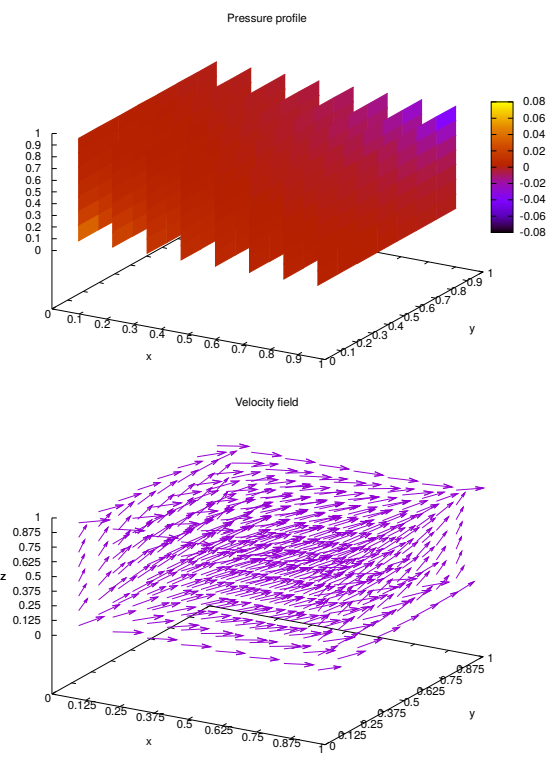

Figure 2. Pressure (top) and velocity (bottom) fields.

\section{Conclusions}

In this work, we presented our preliminary numerical simulation for a 3D $1 / 4$ of five spot problem with one injection well and one production well. The quality of the numerical solution is satisfactory and resembles main features of the Darcy flow as expected. We consider homogeneous permeability field and we expect to adress the heterogeneous. We also expect to extend this technique to multiphase flows.

\section{Acknowledgement}

All authors thanks PETROBRAS, CNPq and FAPESP.

1 J. Douglas Jr., P. J. Paes Leme, J. E. Roberts, and Junping Wang, Numer. Math. 65, 95-108 (1993).

2 J. Douglas Jr, F. Furtado e F. Pereira, Computational Geosciences 1.2 (1997).

${ }^{3}$ E. Abreu, A. Alvarez, P. Ferraz e W. Lambert, International Conference on

Approximation Methods and Numerical Modelling in Environment and

Natural Resources MAMERN VI. 2015. 